\title{
Internacionalización del turismo y desarrollo capitalista en América Latina
}

\author{
Cristina Thalasselis \\ Universidad Provincial de Córdoba (UPC). \\ thalasseliscristina@gmail.com \\ https://orcid.org/0000-0002-1 140-6669
}

\author{
How to cite this paper: \\ Thalasselis, Cristina (2020). Internacionalización del \\ turismo y desarrollo capitalista en América Latina. \\ Revista Encuentros, Universidad Autónoma del Caribe. Vol. 18- \\ 3. Dossier. \\ Doi: 10.15665/encuent.v18i3.2131
}

Recibido: 25 de septiembre de 2019 / Aceptado: 5 de enero de 2020

\section{RESUMEN}

La expansión del turismo internacional como estrategia de desarrollo socioeconómico es producto de procesos históricos complejos, cuya comprensión puede resultar de valor para analizar las posibilidades y los problemas relativos a su desenvolvimiento. El presente trabajo explora los procesos de internacionalización del turismo iniciados en los años de la Segunda Posguerra, considerando las condiciones y las lógicas bajo las cuales se produce la integración de la región latinoamericana en la estructura mundial del turismo. Se propone que el análisis crítico del papel del turismo internacional en el desarrollo latinoamericano requiere volver la mirada sobre este periodo clave, puesto que es entonces cuando, a partir de la concomitancia entre mundialización del turismo, modernización capitalista e ideología del desarrollo, se configuran las premisas fundantes de una concepción de desarrollo turístico cuyas notas esenciales gravitan hasta el presente.

Palabras clave: internacionalización - turismo - capitalismo - desarrollo - América Latina

\section{Internationalization of Tourism and Capitalist Development in Latin America}

\begin{abstract}
The expansion of international tourism as a socio-economic development strategy is the product of complex historical processes, whose understanding can be valuable to analyze the possibilities and problems related to its development. The present work explores the processes of internationalization of tourism initiated in the years of the Second Postwar period, considering the conditions and logics under which the integration of the Latin American region in the world structure of tourism takes place. It is proposed that the critical analysis of the role of international tourism in Latin American development requires a look in this key period, since it is then that, based on the concomitance between the globalization of tourism, capitalist modernization and development ideology, foundational premises of a conception of tourism development are formed whose essential notes gravitate up to the present.
\end{abstract}

Key words: internationalization - tourism - capitalism - development - Latin America 


\section{INTRODUCCIÓN}

El turismo se ha transformado en uno de los sectores económicos de mayor crecimiento a escala planetaria. En América Latina, numerosos gobiernos le han asignado un papel clave como impulsor del desarrollo socioeconómico, incentivando su inclusión prioritaria como asunto de política pública. La integración de la región latinoamericana en la estructura mundial del turismo se remonta al periodo de agotamiento del proceso de industrialización sustitutiva de importaciones imperante hasta fines de los años sesenta y se inscribe en el marco de la necesidad de concebir e implementar una nueva línea estratégica o patrón de desarrollo. En aquellos años, las instituciones económicas mundiales comienzan a atribuir gran importancia a las corrientes turísticas internacionales, sosteniendo que generan ingresos monetarios que contribuyen a reducir el déficit estructural de la balanza de pagos de las naciones subdesarrolladas. Uno de los aspectos más notorios que se desprende de las argumentaciones de la época, es el cambio de sentido producido en la interpretación de la función del turismo, puesto que el mismo deja de ser considerado preponderantemente como una práctica que es consecuencia natural de cierto grado de desarrollo socioeconómico, para convertirse en causa del mismo.

El vínculo entre turismo y desarrollo, gestado en el marco de los procesos de internacionalización turística, ha sido analizado problematizando las lógicas que enlazan a las economías desarrolladas con el desarrollo económico y social de los países subdesarrollados (Lanfant, 1980). Estos análisis han sido retomados en el plano regional, revelando la intervención de las instituciones internacionales de asistencia técnicofinanciera en la determinación de una doctrina de referencia en materia de desarrollo turístico, como así también su incidencia en la gobernabilidad de tales procesos (Capanegra, 2012). Más recientemente, se ha profundizado en la participación e influencias de dichas instituciones mundiales en la planificación del desarrollo turístico latinoamericano (Icaza, 2017). Los estudios referidos sugieren la importancia de considerar las complejidades de los procesos históricos en los que se gesta la concepción del turismo como factor de desarrollo para los países de nuestra región, puesto que las notas esenciales de las estrategias que se imponen en la materia perviven en el presente, sin que se examine en profundidad su mayor o menor adecuación a la realidad existente. En esta dirección, el presente trabajo propone que la adecuada interpretación del papel que desempeña el turismo como factor de desarrollo en América Latina, requiere volver la mirada sobre este periodo germinal, asumiendo una perspectiva relacional que posibilite analizar los complejos procesos emergentes de la concomitancia entre internacionalización del turismo, modernización capitalista e ideología del desarrollo.

En función de estas coordenadas, este artículo presenta, primeramente, una contextualización de la posición relativa deAmérica Latina en el marco de los movimientos turísticos mundiales, señalando una serie de contrastes inherentes a su desenvolvimiento. Seguidamente, se exploran las condiciones regionales y mundiales que posibilitaron los procesos de incorporación del turismo internacional como factor de desarrollo económico en América Latina. A través de la recuperación de aportes de la literatura especializada, e integrando algunas perspectivas de la sociología latinoamericana del desarrollo, se propone una reconstrucción de tales circunstancias. En este contexto, se ponen de relieve los problemas imperantes en la realidad regional de la época, como así también las lógicas bajo las cuales se produce la integración de la región en la estructura mundial del turismo. En un tercer momento, y retomando las perspectivas referidas, se abordan las dimensiones políticas e ideológicas imbricadas en la renovada función que el turismo internacional asume en el sistema económico global. Finalmente, se ofrecen algunas conclusiones tendientes a repensar el papel del turismo en el desarrollo latinoamericano.

\section{A modo de contextualización: América Latina en el marco de los movimientos turísticos mundiales}

El devenir del turismo en el mundo, y en particular en América Latina, se encuentra atravesado por una serie de contrastes. Por una parte, la atención que concentra el crecimiento cuantitativo del sector, acompañada de la proliferación de políticas estatales de desarrollo tejidas en torno a tal cuestión, da cuenta del notorio interés que el mismo ha suscitado como impulsor del desarrollo 
socioeconómico, vinculado a la generación de divisas y empleo $^{56}$. En el ámbito internacional, numerosos organismos le han asignado un papel clave como motor de desarrollo, e incluso como medio tendiente a contribuir a la reducción de la pobreza, incentivando su inclusión prioritaria en el marco de las políticas nacionales e internacionales ${ }^{57}$. A este respecto, no resulta difícil advertir la manera en que tales argumentos se articulan en las políticas estatales que impulsan la expansión de la industria de los viajes en los países de nuestra región ${ }^{58}$.

Sin embargo, una mirada focalizada en torno a la posición del turismo latinoamericano en el contexto mundial evidencia que la participación regional en el reparto de los beneficios generados por el turismo internacional es aún muy débil. En este sentido, es notoria la concentración tanto de los flujos turísticos como de sus repercusiones económicas en las regiones de Europa, América del Norte y los países de Asia y el Pacífico. Estas superficies condensaron en el año 2017 casi el 87 por ciento de los ingresos en concepto de turismo, frente al 5,6 por ciento alcanzado de manera conjunta por los destinos de América del Sur, América Central y el Caribe. Además, si se circunscribe la mirada al continente Americano, se observa que durante el año 2017 se registraron 210,9 millones de arribos turísticos -el equivalente al 16 por

56 De acuerdo a estadísticas procesadas por la Organización Mundial del Turismo (OMT) -agencia especializada de las Naciones Unidas en la materia- en el año 1950 las llegadas de turistas internacionales en el mundo alcanzaron los 25 millones, cifra que creció a 1.326 millones en 2017. En lo que respecta a los ingresos turísticos mundiales, los mismos han ascendido de 2.000 millones de dólares en 1950 a 1.340 .000 millones en 2017 (Panorama OMT del turismo internacional, Ediciones 2017 y 2018). En el documento que condensa el panorama estadístico del turismo internacional correspondiente al año 2017, el referido organismo ha señalado que, como categoría mundial de exportación, el turismo ocupa el tercer lugar por detrás de los productos químicos y de los combustibles, y por delante de la automoción y de la alimentación. En la misma publicación, se informa que uno de cada diez puestos de trabajo en el mundo están respaldados por el sector turístico.

57 Son muy diversos los organismos internacionales se han sumado a la cooperación en el sector turístico. Getino (2009) precisa que entre ellos figuran las Naciones Unidas y algunos de sus programas más relevantes, como así también el Fondo Monetario Internacional (FMI), la Organización para la Cooperación y el Desarrollo Económico (OCDE), y el Banco Interamericano de Desarrollo (BID). Además, es pertinente destacar la participación del Banco Mundial como una de las entidades de financiamiento internacional más conocidas (Molina, 2005). En lo que respecta a la vinculación entre el turismo y la reducción de la pobreza, puede consultarse el trabajo de Gascón (2011).

58 La OMT impulsa la integración sistemática del turismo en la agenda global y su inclusión prioritaria en las políticas nacionales e internacionales, fundadas en el valor que tal organismo atribuye al sector turístico como motor de crecimiento socioeconómico y de desarrollo. ciento del total mundial- y 326,2 mil millones de dólares por ingresos en concepto de turismo internacional, cifra que representa el 24,3 por ciento de dicho total ${ }^{59}$. En este contexto, los Estados Unidos y Canadá concentraron 97,74 millones de llegadas, lo que equivale al 46,5\% del total continental y 231,08 mil millones de dólares en materia de ingresos, cifra que significa el $70,8 \%$ del total para América. En el reparto regional de los ingresos por turismo internacional, la posición más destacada corresponde a los Estados Unidos, país que concentra el $64,6 \%$ del total continental. El resto del continente tuvo una representación conjunta del 53,5 por ciento de los arribos y del 29,2 por ciento de los ingresos.

Más allá de la elocuencia de estas cifras, cabe señalar que la progresión de la afluencia turística internacional en la región latinoamericana, aunque débil en el contexto de los términos antes referidos, es creciente y sostenida. Aun así, la relación entre las llegadas de turistas y los beneficios que las mismas generan en los destinos receptores es un aspecto que merece ser indagado con mayor detenimiento, puesto que el incremento en el número de arribos no garantiza necesariamente un impacto económico equivalente. El turismo es un fenómeno complejo. Si bien en el ámbito mundial genera millones de desplazamientos y ostensibles repercusiones económicas, también supone la integración de sociedades muy disímiles a las reglas del juego y a las relaciones implícitas en un modelo de desarrollo que, con diversos matices, involucra condiciones desiguales de comercio y de poder, intereses del capital extranjero y local, fuerte participación de compañías multinacionales, creciente concentración empresarial, transferencia de modelos culturales, mercantilización de productos fundados en los atractivos naturales y culturales de los países receptores y, en suma, la adaptación de una serie de valores universales del desarrollo a las condiciones singulares de cada realidad nacional ${ }^{60}$.

Ahora bien, ¿cómo fue que comenzó a concebirse la idea de que la expansión del turismo internacional podía constituirse en una alternativa viable de desarrollo? Y

59 Publicación anual: Panorama OMT del turismo internacional. Edición 2018.

60 Sobre las tensiones y contradicciones vinculadas a los procesos de expansión del turismo pueden consultarse los trabajos de De Kadt (1979), Nash (1992); y más recientemente los de Buades, Cañada y Gascón (2012) y Dachary, Arnaiz Burne y César Arnaiz (2018), entre otros. 
en conexión con ello, ¿cuáles fueron las condiciones regionales e internacionales que posibilitaron su incorporación como factor de desarrollo para nuestras sociedades? En el siguiente apartado, se exploran las respuestas a estos interrogantes.

\section{El turismo internacional como factor de desarrollo en América Latina}

Aunque los orígenes del turismo se remontan a comienzos del siglo XIX, en coincidencia con el desarrollo y la consolidación del capitalismo industrial, su emergencia como modelo masivo tiene lugar a partir de mediados del siglo XX (Hobsbawm, 2006). Este periodo posterior a la Segunda Guerra Mundial, motorizado por la revolución científico técnica y signado por una serie de reconversiones económicas, sociales, políticas y culturales, reviste aquí particular interés, puesto que representa el momento histórico en el que cobra mayor fuerza el vínculo entre turismo y desarrollo, al tiempo que se configura la ideología y el modelo que se propagaría, desde entonces, en América Latina.

En efecto, en aquellos años de posguerra, la necesidad de relanzar la economía capitalista mundial, de reconstruir los países que habían sido escenario del conflicto, de impedir el avance comunista y de implementar programas de asistencia al denominado tercer mundo, determinaron que el desarrollo cobrara fuerte centralidad en las agendas internacionales, inaugurando, bajo la influencia directa de los Estados Unidos y Europa, una etapa en la que aquel objetivo logró presentarse como un horizonte alcanzable para los países de la periferia (Madoery, 2016). En lo que respecta a la situación latinoamericana de estos años, la preocupación central de la CEPAL (Comisión Económica para América Latina y el Caribe) giraba en torno a las desigualdades entre economías nacionales que se venían acentuando a través del comercio internacional. Más específicamente, se advertía que existía una caída de los precios de los productos primarios, en relación a los precios de los productos industriales exportados por el centro, problema que se traducía en un deterioro constante de los términos de intercambio, cuyos efectos comenzaban a impactar severamente en los países de la región (Prebisch, 1963). Muchos de los análisis ponían en duda la importancia del comercio internacional por sí mismo como fundamento de la igualdad económica entre naciones e incorporaban factores institucionales y estructurales a la explicación de tal desigualdad, pero sin cuestionar la posibilidad y la deseabilidad de alcanzar los mismos patrones de desarrollo de los países industrializados (Cardoso, 1981).

$\mathrm{Al}$ mismo tiempo, en el ámbito mundial, los avances tecnológicos, el desarrollo de la industria aeronáutica y el crecimiento de las empresas turísticas norteamericanas comenzaban a anticipar el despegue del turismo como modelo masivo ${ }^{61}$. Estados Unidos había reconocido prontamente el potencial que presentaba tal sector como medio de ayuda económica y como instrumento ideológico de política exterior, sobre todo para los países que experimentaban progresivas dificultades para incorporarse a un comercio internacional de creciente competencia y menores posibilidades para obtener las divisas necesarias para el desarrollo. En este contexto, el auxilio al mundo subdesarrollado apuntaba a una mejora de su desventajosa situación con el fin de reducir el riesgo potencial de radicalización de las posturas ideológicas y de eventuales intentos de cambio de las estructuras (Jiménez, 1993). Así, en la coyuntura mundial de los años 60, mientras que un número cada vez mayor de habitantes de las sociedades industrializadas alcanzaba la posibilidad de viajar, el turismo asumía el estatuto de fenómeno de masas ligado a la cooperación internacional y a su difusión como actividad beneficiosa para los países subdesarrollados.

En lo que respecta a la progresión de los desplazamientos, es ilustrativo señalar que entre los años 1950 y 1970 la cifra mundial de desplazamientos de turistas internacionales pasó de 25,28 millones a 159,69 millones $^{62}$. Este veloz desarrollo se asentó en dos factores principales: por una parte, la rápida acumulación por parte de las naciones centrales de cuantiosos recursos económicos producto de la situación interna imperante en cada país y, por otra parte, la creación a nivel transnacional de redes empresariales en las que convergieron grandes

61 En este contexto se produce el desarrollo acelerado de la era del jet, que duplicaría por dos la velocidad de vuelo, y la aparición de las aeronaves de gran capacidad (Getino, 2009). En conexión con ello, se destaca la irrupción de los tour operadores en el mercado turístico con el fin de maximizar la eficiencia de la comercialización, acompañada de la expansión de importantes corporaciones de hoteles norteamericanos (Jiménez, 1993).

62 Estadísticas de la Organización Mundial del Turismo referidas a llegadas de turistas internacionales en el mundo (1950-1990), en Jiménez (1993). 
compañías aéreas, cadenas de hoteles, poderosos grupos bancarios, agencias mundiales de viajes, empresas internacionales de renta de automóviles y medios masivos de promoción y publicidad ${ }^{63}$. Para los países de menor desarrollo, el turismo representaba una alternativa a la fracasada o imposible industrialización, en tanto que para los países centrales se presentaba como una opción de modernización y aprovechamiento máximo de los recursos (Dachary, Arnaiz Burne y César Arnaiz, 2018).

Un hecho sobresaliente que explica la institución y propagación de tal modelo a escala global es la decidida intervención en la difusión del turismo de un conjunto de instituciones mundiales -Fondo Monetario Internacional, Organización Mundial del Turismo, Banco Mundial, Banco Interamericano de Desarrollo, entre otras-, las cuales asignan gran importancia a las repercusiones económicas generadas por los flujos turísticos internacionales (Lanfant, 1980). Reparar en esta intervención resulta relevante, puesto que hace posible identificar una doble operación. Por una parte, desde ese lugar se define el marco conceptual, interpretativo e ideológico que concibe al turismo como factor de desarrollo para las naciones periféricas, puesto que se aduce que los efectos producidos por tal sector, principalmente internacional, generarían notables beneficios en materia de comercio exterior, contribuyendo a reducir el déficit estructural de la balanza de pagos de las naciones subdesarrolladas y, por efecto derrame, mitigaría la pobreza. A este respecto, Marie Francoise Lanfant (1980), en su esclarecedor estudio acerca del turismo en el proceso de internacionalización, precisa que en el año 1963 las Naciones Unidas hicieron suya esta tesis, señalando que el turismo ofrece la posibilidad de aportar, y aporta efectivamente, una contribución vital al crecimiento económico de los países subdesarrollados.

Así, una vez establecido el ideal de desarrollo a alcanzar y configurado el modelo turístico de validez universal que contribuiría a hacerlo posible, el paso a la segunda operación estaba dado. En esta dirección, los países en desarrollo fueron incentivados, a partir de una intensa campaña de persuasión, a abrir sus fronteras al sector turístico, atribuyéndole una posición

63 Sobre este punto, ver Getino (1991) y Moreno (1971). Este último autor señala la tendencia a la centralización del negocio turístico en grandes sistemas internacionales controlados principalmente por conglomerados norteamericanos. prioritaria en sus economías y recibiendo a los capitales extranjeros interesados en invertir en esta esfera, lo cual suponía concederles ventajas fiscales y garantías (Lanfant, 1980). En consecuencia, un buen número de países del Tercer Mundo se lanzaron, de la mano de la asistencia financiera internacional, a la realización de grandes proyectos turísticos en el marco de un modelo de planificación dictado por los parámetros de la demanda de las poblaciones más ricas y sometido a las condiciones operacionales de la estructura funcional del turismo internacional (Jiménez, 1993). En la región latinoamericana, numerosos gobiernos orientaron sus esfuerzos al desarrollo del turismo receptivo a la espera de obtener los beneficios que generaría el modelo promovido. Sin embargo, en muchos casos la situación de dependencia vinculada a la participación de poderosas empresas transnacionales extranjeras en el manejo de los flujos turísticos y en la captación de divisas provocó una importante merma del derrame económico que se suponía proporcionaría este sector exportador. Con respecto al devenir de tales empresas, se ha señalado que solamente en México existían, en 1980, 63 corporaciones con participación extranjera entre operadores de viajes, hoteles y restaurantes, de las cuales aproximadamente las dos terceras partes (63 por ciento) contaban con capital extranjero superior al 40 por ciento ${ }^{64}$. En conexión con ello, es pertinente señalar que la creciente importancia del turismo a partir de la Segunda Guerra Mundial provocó que los países hegemónicos, inicialmente Estados Unidos y Gran Bretaña, intentaran frenar la fuga de divisas originada por la salida anual de millones de turistas adoptando la estrategia de ir a buscar el dinero allí donde se gasta, para lo cual la constitución de empresas multinacionales funcionó como estrategia tendiente a crear un circuito cerrado que garantizara el retorno incrementado de las divisas que salían de sus países (Getino, 1991).

En lo que concierne a la asistencia técnica y financiera, cabe precisar que hacia 1969, el Banco Interamericano de Desarrollo interviene en Latinoamérica para financiar obras de infraestructura, instalaciones básicas -principalmente hoteles- y atracciones culturales para el turismo. El primer proyecto concebido y financiado por tal entidad fue Cancún en México, al que le siguieron el proyecto de desarrollo integrado del área

64 Ver Molina (2005). 
de Machu Pichu-Cuzco-Puno en Perú, el proyecto de desarrollo turístico de Panamá y el proyecto de Bahías de Huatulco en México (Altés, 2006). Además, durante este período, el Banco Mundial también aprobó una serie de proyectos orientados en la misma dirección y localizados en Jamaica, El Salvador, Colombia, Panamá y México (Jiménez, 1993). Un rasgo característico de la entrada de inversiones del capital internacional durante las décadas de los 50 y 60, consistió en la necesidad de creación de infraestructura que soportara su instalación, en función de lo cual se buscó apoyo en el ahorro interno de los países donde dichas inversiones tenían lugar. Así, y en función de este esquema, quedó configurada una lógica en virtud de la cual a los países receptores les correspondía afectar recursos internos para garantizar una amplia infraestructura que viabilizara el ingreso y el establecimiento masivo del capital extranjero (Dos Santos, 1996).

El modelo de desarrollo turístico que tuvo lugar en esta época, fundado en aspectos cuantitativos y en la preeminencia de poderosas empresas transnacionales, no tardó en despertar serios cuestionamientos debido a los altos costos que significaba esta relación desigual traducida en evidentes impactos negativos sobre el territorio, la naturaleza y las comunidades receptoras ${ }^{65}$. Antes de este periodo de expansión, el turismo no estuvo ausente como cuestión de política pública en Latinoamérica sino que, por el contrario, jugó distintos papeles en el marco de los procesos políticos, sociales y económicos que atravesaron nuestra región. Así, por ejemplo, en los gobiernos de Getulio Vargas en Brasil (1937-1954) y de Juan Domingo Perón en Argentina (1946-1952), la actividad turística se presentó inserta en el marco de nuevas conquistas sociales, al tiempo que sirvió como estrategia de acumulación de poder y como agente reactivador de economías (Comparato, 2014). Otros países latinoamericanos, cada uno con sus especificidades, también fueron otorgando importancia a esta actividad. En el caso de Perú, por ejemplo, el accionar del Estado, sumado a la temprana relevancia que comenzó a asumir el turismo interno y al interés de las élites locales en el desenvolvimiento de esta actividad generaron que entre 1930 y 1940, ya existieran

65 Jafari (2005) señala la emergencia, durante la década de los años 70, de una plataforma de estudios críticos preocupados por la protección de las culturas y la naturaleza frente a diversos aspectos e influencias de la expansión del turismo. acciones significativas en materia de promoción del turismo receptivo e interno (Armas Asín, 2018). Las acciones referidas pueden situarse como parte de una etapa de turismo industrial temprano originada en el siglo XIX y extendida hasta los inicios de la Segunda Guerra Mundial, que para el caso de América Latina comprende, entre otras manifestaciones, la aparición de los primeros destinos turísticos, la expansión del transporte de superficie y la creación de las primeras oficinas gubernamentales de turismo (Molina, 2000).

Aunque la cuestión turística ya había sido incluida en el arco de los asuntos de Estado de los países de la región, y por lo tanto comenzaba a asumir diversas orientaciones como estrategia de política pública, el turismo desarrollado a partir de la década de los años 60 presenta la particularidad de introducir una certeza en función de la cual se instituye y difunde la concepción mundial dominante: el turismo, sobre todo internacional, es un hecho económico relevante para las sociedades subdesarrolladas. Dicha formulación supone la entrada en juego de las diferentes sociedades nacionales en un proceso de internacionalización que las vincula a sistemas económicos, sociales y culturales organizados a escala mundial, desintegrando o soslayando los sistemas de referencias nacionales (Lanfant, 1980). Del mismo modo, las políticas de desarrollo asumidas por los países en los que el turismo desempeña un papel económico fundamental, incrementan su sensibilidad a la presión internacional propia de la estructura funcional del turismo (Moreno, 1971). En conexión con ello, se pone en evidencia un alto grado de sujeción de las economías de las sociedades receptoras a los vaivenes de los flujos turísticos en función de las percepciones de la demanda frente a los cambios políticos, económicos y sociales del contexto mundial que prevalece (Jiménez, 1993).

La reconstrucción esbozada hasta aquí, posibilita advertir algunas de las principales condiciones históricas concretas (económicas, sociales, culturales, políticas e ideológicas) que se imbrican en los procesos de internacionalización del turismo y en su difusión como factor de desarrollo económico para los países de nuestra región. Buena parte de la complejidad de tales procesos estriba en el hecho de que los mismos no se producen en el vacío, sino en el marco de un sistema mundial constituido por relaciones efectivas de poder. En el análisis de este contexto problemático, 
las condiciones y relaciones internas no bastan para explicar la compleja dinámica socio histórica, sin embargo, tampoco resulta suficiente asumir la primacía de las condiciones y relaciones externas, puesto que ello conduciría a prescindir de las mediaciones existentes entre tales determinaciones y los movimientos y las transformaciones de las sociedades latinoamericanas.

A partir de estas coordenadas, y sin pretender aprehender todos los aspectos y matices intervinientes en el marco de las discusiones que desde allí se desprenden, en los siguientes apartados se abordan dos dimensiones que se consideran relevantes para los objetivos de este trabajo. Por una parte, la referencia a los principales problemas relativos a los procesos de desarrollo en la región latinoamericana en los años en que el turismo internacional se presenta como estrategia de desenvolvimiento económico. Desde este lugar, se procura una aproximación a las condiciones y a las lógicas intervinientes en la integración regional en la estructura mundial del turismo. Por otra parte, en estrecha vinculación con ello, se abordan las implicaciones ideológicas y políticas que subyacen a la función que el turismo comienza a asumir en el sistema económico mundial.

\section{La integración de la región latinoameri- cana en la estructura mundial del turismo}

Como se ha señalado anteriormente, la integración de la región latinoamericana en la estructura mundial del turismo tiene lugar en el periodo de agotamiento del proceso de industrialización sustitutiva de importaciones (ISI) imperante hasta fines de los años sesenta, inscribiéndose en el marco de la necesidad de concebir e implementar un nuevo patrón de desarrollo. Hasta entonces, los intentos efectuados por generar las condiciones necesarias para para la integración nacional de la economía y para una rápida industrialización, no habían logrado garantizar los requisitos básicos asociados a una dinámica de crecimiento sobre bases autónomas (Prebisch, 1963). En estos años, no sólo persisten las dificultades que la política de desarrollo centrada en torno a la ISI había intentado solucionar sino que, además, se añaden nuevos problemas, tales como el creciente endeudamiento exterior, el desequilibrio continuo de la balanza comercial y las presiones inflacionarias.
Tres rasgos estructurales preeminentes configuran la compleja realidad regional de la época: la marcada concentración del ingreso en algunos estratos privilegiados, las condiciones de exclusión de una amplia fracción de la población nacional del orden económico, social y político existente, y la coexistencia de estructuras económicas y socioculturales en diferentes épocas históricas (Fernandes, 2008). Este último aspecto involucra la presencia simultánea de elementos de modernidad y de elementos tradicionales en las estructuras de la región, particularidad que da cuenta de una cierta porosidad en función de la cual dichas estructuras logran absorber algunos rasgos modernos, sin que ello implique el abandono de los patrones tradicionales (Medina, 1963). En este escenario, fuertemente signado por los problemas vinculados a la dependencia de los países latinoamericanos de los mercados internacionales, las discusiones relativas a la explicación del subdesarrollo y a la definición de estrategias comienzan a trascender el ámbito restringido de la industrialización y las políticas de estímulo a la misma, para ampliarse a la totalidad de relaciones, dinámicas y estructuras que influyen en el proceso de desarrollo.

Aunque se han esbozado de manera muy sintética, las referencias precedentes posibilitan efectuar algunos señalamientos de interés en torno a las condiciones en que los países latinoamericanos ingresan en la estructura mundial del turismo, como así también en lo concerniente a las lógicas que subyacen a tal integración. En esta dirección, al menos tres puntos resultan relevantes. En primer lugar, se advierte que el vínculo entre turismo y desarrollo, gestado en el marco de los procesos de internacionalización, se funda en argumentos que recogen los problemas imperantes en la realidad regional de la época, articulándolos en un planteamiento que asocia el impacto económico directo del turismo con la posibilidad de acortar la brecha que separa al mundo subdesarrollado de las sociedades más ricas. En esencia, esta lógica implica considerar que tales impactos -fundamentalmente la generación de divisas y empleo- constituyen, por sí solos, una garantía de desarrollo, soslayando por lo menos dos cuestiones centrales en el contexto de los históricos desafíos que enfrenta la región. Por una parte, la pregunta acerca de la medida en que los efectos económicos generados por 
la afluencia turística internacional tienden a alcanzar a la población en su totalidad o, por el contrario, propenden a concentrarse en algunos sectores específicos. Por otra parte, pero también en conexión con lo anterior, el cuestionamiento relativo al balance entre la carga de la acumulación del capital que soportan los países latinoamericanos y la apropiación de las cuotas del excedente económico por parte de las economías centrales. Estos interrogantes ubican en el centro del debate el problema de la retención y distribución de los ingresos producidos por el turismo en la región, puesto que, si bien el impulso de dicha actividad se justifica en función de indicadores cuantitativos referidos a sus efectos en la balanza de pagos, el producto bruto nacional y el empleo general, resultan inciertos los modos concretos en que tales indicadores se relacionan con los fines últimos del desarrollo.

En segundo lugar, y como se ha referido anteriormente, dado que la incorporación de los países de la región latinoamericana en la estructura mundial del turismo se efectúa en una posición dependiente -puesto que ninguno de ellos posee una economía homogénea, ni tampoco potencialidades organizadas de desarrollo autónomo efectivo- la situación de subordinación al mercado mundial demanda, de modo continuo, la realización de ajustes estructurales que privilegian la lógica de las relaciones exteriores. En vinculación con ello, es importante advertir que la satisfacción de los requerimientos de la demanda turística internacional supone la implementación de una serie de transformaciones que profundizan la dependencia de los países de la región con respecto a los bienes y mano de obra especializada de importación. Uno de los rasgos específicos de la acelerada expansión del turismo internacional en la época consiste en el hecho de que el mismo no admite un mejoramiento gradual de los servicios, simultáneo al desarrollo gradual del país, razón por la cual su desenvolvimiento se presenta inevitablemente ligado a la implantación masiva de tecnología e instituciones, como así también a la provisión externa de capital y de control financiero.

En conexión con lo anterior, y en tercer lugar, es preciso reconocer que el desarrollo del turismo internacional, tal como ocurre en el contexto de los procesos de mundialización, supone la existencia de determinados patrones de asociación entre el capital estatal, el capital privado local y el capital transnacional. En este sentido, comprender los alcances y las implicancias de la modalidad de acumulación transnacionalizada requiere explorar la constitución de aquellas formas que viabilizan su expansión en la región, como así también indagar las tensiones entre los imperativos de la acumulación de carácter transnacional y las posibilidades de un desarrollo nacional no dominado por las estrategias de tales empresas.

Es frecuente que se acepte con carácter dogmático una supuesta correlación entre el ingreso de flujos turísticos internacionales y el desarrollo socioeconómico de los países receptores. Esta lógica ha impulsado los procesos que hasta aquí se han presentado. En muchos casos, continúa impulsando en el presente las políticas que se tejen en torno tal cuestión en América Latina. Sin negar que dicha correlación puede darse en determinadas circunstancias, tampoco cabe dudar de que la historia y el análisis de los procesos de expansión del turismo internacional advierten acerca de la fragilidad de tal planteamiento, cuando no permiten ponerlo en cuestión. En este sentido, si bien en aquellos años el turismo se presenta como un factor de desarrollo tendiente a acortar la brecha entre los países centrales y las sociedades menos desarrolladas, su expansión en lo real supone la reproducción de relaciones asimétricas de intercambio entre formaciones socioeconómicas diferentes, articuladas en función del mercado mundial. Según se ha visto, dicha articulación involucra una serie de condiciones de subordinación de las sociedades periféricas a las exigencias de tal mercado, requiriendo, principalmente, la realización de una variedad de ajustes estructurales en línea con las demandas de los países centrales.

A este respecto, posiblemente uno de los principales núcleos problemáticos del planteamiento que hace del turismo internacional un factor de desarrollo para los países del denominado Tercer Mundo, consista en la compleja naturaleza de los intercambios entre formaciones capitalistas centrales y periféricas, como así también en el carácter de los nexos de interdependencia generados entre ambas, con todo lo que ello conlleva en términos de desigualdad. Como es evidente, profundizar en el análisis de la función que asume el turismo en el sistema económico global requiere, inevitablemente, considerar el condicionamiento decisivo que suponen 
las relaciones efectivas de poder y la dirección que asumen sus configuraciones concretas en el contexto de aquel orden mundial.

\section{La dimensión político-ideológica del modelo de turismo internacional}

Según se ha referido hasta aquí, la configuración del turismo internacional como factor de desarrollo para los países subdesarrollados, se gesta en el marco de procesos históricos mundiales, de naturaleza socioeconómica y sociocultural. Sin embargo, lo que interesa postular en esta instancia, es la influencia decisiva que supone su ingreso como estrategia de política exterior.

En esta dirección, es preciso tener presente que en la constelación histórica de los años de la Segunda Posguerra, época en la que cobran fuerza los procesos de internacionalización del turismo, lo que verdaderamente se encuentra en juego es una férrea defensa del capitalismo privado por parte de las naciones capitalistas avanzadas de Europa, América y Asia. En aquel clima político, en el que el conflicto entre los países socialistas y los países capitalistas ocupa el lugar central en la historia, se produce la irrupción de un renovado proceso de modernización que estimula el crecimiento económico de los países latinoamericanos, impidiendo, a la vez, su autonomía real. Uno de los rasgos más característicos y trascendentales de aquel proceso consiste en el hecho de que su influencia no se limita a los aspectos económicos sino que implica, en la práctica, una reconstrucción de las economías, las sociedades y las culturas de nuestra región de acuerdo con los intereses y valores políticos imperantes (Fernandes, 2008).

En el contexto de lo expuesto, interesa subrayar que la comprensión de los fenómenos de interdependencia a escala mundial, característicos de estos años, resulta inescindible de la lógica de los sistemas que vehiculizan su expansión. En este sentido, si bien en los procesos de mundialización capitalista la dimensión económica asume un rol preponderante, no resultan menos importantes las dimensiones políticas que determinan los modos de inserción de las diversas sociedades en el sistema mundial. De allí que sólo en el marco de las tensiones entre aquellas fuerzas políticas resulte posible comprender cabalmente uno de los rasgos fundamentales de este periodo, consistente en la potencia y el dominio que cobra el discurso del desarrollo en esta fase de expansión del capitalismo mundial.

Lo referido anteriormente demarca los ejes fundamentales partir de los cuales es posible pensar en las dimensiones políticas e ideológicas imbricadas en la renovada función que asume el turismo en el sistema económico mundial. En términos concretos, la internacionalización del turismo producida en el marco de los procesos de modernización capitalista iniciados a mediados del siglo pasado, involucra las siguientes manifestaciones: a) la irrupción de corporaciones turísticas norteamericanas o europeas que parecían corresponder a las aspiraciones latinoamericanas de crecimiento nacional autosostenido; b) la influencia ejercida, vía efecto demostración, tanto por las referidas corporaciones como por los flujos turísticos internacionales en la definición de las aspiraciones ideales con relación al futuro y al estilo de vida deseable; y c) la incorporación dependiente de las sociedades latinoamericanas al sistema económico, social y cultural mundial por medio de su ingreso en la estructura internacional del turismo.

El turismo internacional se difunde en un clima político en el que el desarrollo es considerado posible en virtud de la interdependencia en el seno de la economía mundial. En este contexto, el afán de las naciones subdesarrolladas por desenvolver y diversificar sus fuerzas productivas, ligado a la difusión de modelos técnicos de aparente neutralidad y a la convicción de que los problemas inherentes a los intercambios en el sistema capitalista no representan contradicciones radicales sino conflictos momentáneos, se constituyen en aspectos que admiten ser examinados en el marco de los factores que propician la viabilidad de este modelo. Si bien el turismo no nace ligado al problema del desarrollo socioeconómico, cobra tal función en el marco de la reconversión de un sistema capitalista que debió adaptar rápidamente sus tendencias frente al conflicto suscitado por el movimiento de las fuerzas políticas del entonces denominado Tercer Mundo.

Lo anterior no fue sin consecuencias. La mundialización del turismo, iniciada luego de la Segunda Guerra Mundial con la multiplicación exponencial de los desplazamientos, trae aparejada una irrefrenable penetración de tal fenómeno en el tejido económico, 
social y cultural de regiones cada vez más amplias del planeta. Especialmente después de que las Naciones Unidas impulsaran expresamente la expansión del turismo internacional en la Conferencia de Roma de 1963, el fenómeno turístico se ha caracterizado por la combinación de una serie de rasgos específicos: a) un crecimiento a escala planetaria sin precedentes - que le ha valido su actual posicionamiento como tercera categoría de exportación global-, b) una marcada tendencia a la homogeneización internacional por medio de dispositivos de estandarización emanados de la tecnoestructura mundial de coordinación del sector, у c) una aparente neutralidad axiológica que lo ubicaría por fuera de cualquier enfrentamiento ideológico u orientación política.

Sin embargo, considerando lo expuesto anteriormente, y sobre todo si se tiene en cuenta la magnitud económica que denota la industria del turismo, resulta evidente que el modelo de desarrollo imperante no se configura en el vacío, sino en el marco de un sistema mundial constituido por relaciones efectivas de poder. Excluir la dimensión político-ideológica de los análisis para centrarse solamente en el reducto económico, conlleva el riesgo de obturar la comprensión de las repercusiones que los enfrentamientos de intereses en el campo internacional suscitan en la historia y situación de los diversos países de la región. En lo que respecta al devenir del turismo, dichos efectos van mucho más allá de los habitualmente reconocidos.

\section{Consideraciones finales}

La expresión internacionalización del turismo es utilizada, de modo general, para designar los procesos relativos a la masificación de los desplazamientos turísticos internacionales iniciados a partir de mediados del siglo pasado. Dicho enunciado también se emplea para aludir a los fenómenos de interdependencia a escala mundial de las sociedades comprendidas en el marco de aquellas movilidades. Sin embargo, no es frecuente que la expresión se relacione con las lógicas de expansión del capitalismo, y menos aún con las dimensiones políticas e ideológicas de su despliegue. Precisamente a estos últimos aspectos se ha intentado referir en las secciones anteriores del presente trabajo. Al final de este recorrido, y antes de esbozar algunas propuestas de reflexión, se estima oportuno retomar brevemente tres consideraciones centrales.

En primer lugar, la reconstrucción de los procesos de incorporación del turismo internacional como factor de desarrollo económico en América Latina, conduce a examinar la renovada función que dicha actividad asume en el sistema económico global, como así también los agentes y fuerzas que impulsan su expansión. En este sentido, si bien el fenómeno del turismo no nace asociado al problema del desarrollo socioeconómico, en estos años experimenta una notoria reconversión funcional, en virtud de la cual se posiciona y cobra preponderancia dentro de las estrategias a seguir en pos de la modernización y el acortamiento de la brecha que separa a los países centrales de las sociedades menos desarrolladas.

En segundo lugar, la consideración de las condiciones y las lógicas bajo las cuales la región latinoamericana se integra en la estructura mundial del turismo, posibilita advertir la manera en que los problemas imperantes en la realidad regional de la época quedan enlazados en el planteamiento que hace del turismo internacional un factor de desarrollo capaz de contribuir a su resolución. En conexión con ello, los señalamientos efectuados ponen en evidencia las complejidades y contradicciones que conllevan los procesos de expansión del turismo en el mercado mundial. Más específicamente, se destaca como rasgo central la generación de relaciones asimétricas de intercambio entre formaciones socioeconómicas diferentes y, en ese contexto, las condiciones de subordinación que las exigencias de tal mercado suponen para las sociedades periféricas, fundamentalmente en lo relativo a la variedad de ajustes estructurales que requiere su posicionamiento en línea con las demandas de los países centrales. En esta superficie, surgen interrogantes relativos al balance entre la carga de acumulación del capital que soportan los países latinoamericanos y la apropiación de las cuotas del excedente económico por parte de las economías centrales, como así también en lo concerniente a la distribución de los ingresos generados por la afluencia turística internacional, y a los patrones de asociación entre el capital estatal, el capital privado local y el capital transnacional, cuyas formas viabilizan los procesos de expansión del turismo en la región. 
En tercer lugar, la observación de las dimensiones políticas e ideológicas que atraviesan los decisivos acontecimientos históricos de estos años, conduce a situar los procesos de internacionalización del turismo en el marco de la acelerada reconversión capitalista de posguerra, motivada por las tensiones entre los centros hegemónicos y las fuerzas políticas del entonces denominado Tercer Mundo. Uno de los rasgos que particularizan esta fase de expansión del capitalismo mundial, consiste en la potencia y el dominio que cobra el discurso del desarrollo. En este contexto, el turismo internacional se configura y difunde -ligado a instituciones y modelos técnicos de aparente neutralidad- como una estrategia de desarrollo posible en virtud de la interdependencia en el seno de la economía mundial, al tiempo que se presenta como una alternativa a los patrones de desarrollo implementados hasta aquel momento, cuyos problemas traslucen evidentes signos de agotamiento.

Desde entonces, es frecuente que se acepte con un cierto carácter dogmático, una supuesta correlación entre el ingreso de flujos turísticos internacionales y el desarrollo socioeconómico de los países receptores. Sin negar que dicha correlación puede darse en determinadas circunstancias, el análisis de las complejidades inherentes a los procesos de expansión del turismo internacional, advierte acerca de la conveniencia de examinar con cautela tal planteamiento. Aunque con el tiempo se ha avanzado en nuevas formas de abordaje e interpretación del vínculo entre turismo y desarrollo, es notoria la potencia con que las premisas fundantes de aquellos procesos, continúan enraizadas en los objetivos de las políticas públicas que se tejen en torno a tal cuestión, como así también en los argumentos que las sustentan. A este respecto, lo que interesa postular en esta instancia, es que la adecuada valoración de las contribuciones generadas por el turismo internacional a los fines últimos del desarrollo socioeconómico de los países de nuestra región requiere acometer, al menos, dos tipos de análisis.

Por una parte, el abordaje histórico que posibilite entender de qué manera los procesos del desarrollo del turismo han llegado a asumir las características que hoy presentan. Bajo este aspecto, las estructuras de poder en la configuración nacional y en el campo internacional, las relaciones que allí se tejen, las coyunturas externas, y las fuerzas políticas e ideológicas que performan el mundo en el cual los modelos imperantes se vuelven válidos, se presentan como campos de estudio ineludibles.

Por otra parte, pero en estrecha conexión con lo anterior, el segundo tipo de abordaje que aquí se sugiere consiste en el análisis crítico de las lógicas e instrumentaciones relativas al desarrollo del turismo en la especificidad de la realidad latinoamericana. En este contexto, cobra particular relevancia el examen de las configuraciones sociales en las que el turismo se introduce como factor de cambio. Desde dónde se parte, hacia dónde se pretende producir esa transformación, cuáles son sus supuestos y sus condiciones de posibilidad, qué agentes impulsan esos procesos, qué intereses los animan, cómo se enfrentan las resistencias que provoca su establecimiento, qué posición se asume frente a las imposibilidades de lo real y, fundamentalmente, cuál es la concepción de sociedad en juego, son algunos de los interrogantes que es preciso considerar al momento de examinar las proposiciones preeminentes y de pensar otras nuevas.

La institución del turismo internacional como asunto de política pública tendiente al desarrollo no constituye un proceso axiológicamente neutral ni deviene de acontecimientos históricos vaciados de conflictos. Por el contrario, sus lógicas de expansión se gestan en procesos históricos atravesados por luchas de poder, en tanto que sus notas esenciales se configuran en conexión con la racionalidad de los sistemas imperantes. Desmontar estos procesos abre la posibilidad de reconsiderar tanto sus posibilidades como sus límites a la luz de nuevos abordajes. Es probable que este camino contribuya a que los contenidos y las repercusiones económicas, sociales y ambientales de aquellas políticas asuman, también, una orientación diferente.

\section{Bibliografía}

Altés, C. (2006). "El turismo en América Latina y el Caribe y la experiencia del BID”. Serie de informes técnicos del Departamento de Desarrollo Sostenible. Washington D.C.: Banco Interamericano de Desarrollo.

Armas Asín, F. (2018). Una historia del turismo en el Perú. (1 ${ }^{\mathrm{a}}$. ed.). [EPub], Lima: Fondo Editorial Universidad de San Martín de Porres.

Buades, J., Cañada, E. y Gascón, J. (2012). El turismo en el inicio del milenio: una lectura crítica a tres voces. Madrid: Foro de Turismo Responsable.

Capanegra, C. (Septiembre de 2012). Turismo y desarrollo según las Naciones Unidas (1947-1963). $5^{\circ}$ Congreso Latinoamericano de Investigación Turística, San Pablo. 
Encuentros / Internacionalización del turismo y desarrollo capitalista en América Latina

Cardoso, F. (1981). El desarrollo en capilla, en Boletín de Planificación № 12. Santiago de Chile: CEPAL/ILPES.

Comparato, G. (2014). Matices populistas: La política turística de Getúlio Vargas (1937-1954) y de Juan Domingo Perón (1946-1952). Trashumante. Revista Americana de Historia Social. N 3, 116-133.

Dachary, A., Arnaiz Burne, S. y César Arnaiz, F. (2018). Capitalismo, sociedad y turismo. Buenos Aires: Universidad Nacional de Quilmes.

De Kadt, E. (1979). Turismo: ¿Pasaporte al desarrollo? Madrid: Endymion.

Dos Santos, T. (1996). EI desarrollo latinoamericano: pasado, presente y futuro. Un homenaje a André Gunder Frank. Problemas del Desarrollo, Vol. 27, $N^{\circ} 104,143-172$.

Fernandes, F. (2008) [1970]. Patrones de dominación externa en América Latina. En Florestan Fernandes; Heloísa Fernandes (Comp.), Dominación y desigualdad: el dilema social latinoamericano (pp. 113-137). Bogotá: Silgo del Hombre Editores y CLACSO.

Gascón, J. (2011). La metodología "Pro-Poor Tourism": un análisis crítico. Opiniones en desarrollo. Programa Turismo Responsable. Recuperado el 23 de enero de 2019 de https:/www.alainet.org/images/turismo.pdf

Getino, O. (1991). Turismo y desarrollo en América Latina. México D.F.: Limusa.

Getino, O. (2009). Turismo entre el ocio y el neg-ocio: Identidad cultural y desarrollo económico en América Latina y el Mercosur. Buenos Aires: Fundación Centro de Integración, Comunicación, Cultura y Sociedad.

Hobsbawm, E. (2006). Historia del siglo XX. Barcelona: Crítica.

Icaza, C. (2017). Organismos internacionales y políticas turísticas: influencias y relaciones para el caso latinoamericano. En N. Wallingre (Coord.), Desarrollo del turismo en América Latina: fases, enfoques e internacionalización. Buenos Aires: Universidad Nacional de Quilmes., 248-265.

Jafari, J. (2005). El turismo como disciplina científica. En: Política y Sociedad. Vol. 42, $N^{\circ} 1,39-56$

Jiménez, A. (1993). Turismo: estructura y desarrollo. Mexico: Mc Graw - Hill.

Lanfant, M. F. (1980). El turismo en el proceso de internacionalización. En Revista Internacional de Ciencias Sociales. Vol. XXXII, N 1, 14-45.

Madoery, O. (2016). Los desarrollos latinoamericanos y sus controversias. Ushuaia: Ediciones UNTDF.

Medina Echavarría, J. (1963). El desarrollo social de América Latina en la postguerra. Buenos Aires: Solar/ Hachette.

Molina, S. (2000). Conceptualización del turismo. México: Limusa.

Molina, S. (2005). Planificación integral del turismo: un enfoque para Latinoamérica. México: Trillas.

Moreno Toscano, O. (1971). El turismo como factor político en las relaciones internacionales. En Foro Internacional. Vol. XII, 1 (45), 66-94.

Nash, D. (1992). El turismo considerado como una forma de imperialismo. En Valene Smith (Ed), Anfitriones e invitados. Antropología del turismo. Madrid: Endymion, 69-91.

Organización Mundial del Turismo (2017). Panorama OMT del turismo internacional. Edición 2017. Recuperado el 15 de enero de 2019 de https:/ www.e-unwto.org/doi/pdf/10.18111/9789284419043

Organización Mundial del Turismo (2018). Panorama OMT del turismo internacional. Edición 2018. Recuperado el 15 de enero de 2019 de https:// www.e-unwto.org/doi/pdf/10.18111/9789284419890

Prebisch, R. (1963). Hacia una dinámica del desarrollo latinoamericano. México: Fondo de Cultura Económica. 